\title{
La innovación en emprendimientos microempresariales en Palmira, Valle del Cauca
}

\section{Innovation in micro-enterprise entrepreneurships in Palmira, Valle del Cauca}

\author{
Luis Enrique David Tenorio ${ }^{1}$ \\ Natali Cruz González ${ }^{2}$ \\ DOI: 10.29151/hojasyhablas.n18a6
}

Abstract

\begin{abstract}
Resumen
En Colombia, la base de la economía son las microempresas. Estas tienen una participación del 96,1\% (DNP, 2006) del total de las empresas, y su rasgo principal es contar con una planta de personal inferior a 10 colaboradores, siendo generadoras de autoempleo a sus mismos propietarios. Lo expresado anteriormente, puede ser considerado como emprendimientos de subsistencia, liderados por personas con grandes cualidades, pero con desconocimientos sobre temas fundamentales como la creación de empresa y la competitividad. La presente investigación tomó como muestra emprendimientos ubicados en el municipio de Palmira (Valle del Cauca) y facilitó un acercamiento a la situación actual del emprendimiento microempresarial. La información se recolectó por medio de la asesoría realizada a cada emprendedor en la creación y fortalecimiento de su unidad productiva, lo que ayudó a los investigadores a identificar aspectos que no se encuentran por medio de una encuesta o entrevistas. El objetivo fue dar cuenta de las características del emprendimiento microempresarial y su influencia en el proceso de innovación.
\end{abstract}

Palabras clave: Innovación; Empresa; Economía; Asesoría; Competitividad.
The economy in Colombia is based on micro-sized companies, which still make up 96.1 per cent of the total companies (DNP, 2006). Their main characteristic is to have a staff of less than 10 employees and, in many cases, their owners are self-employed by them. These enterprises could be classified as subsistence economies with good human qualities for the people who create them, but without competitiveness. The present research studies enterprises located in Palmira - Valle del Cauca, generating an approach to the current situation of micro business entrepreneurship. The information was collected through the support given by Escuela Nacional del Deporte University, helping the researchers to find aspects that they could not have identified in a survey or interviews. The objective was to identify the characteristics of microenterprise entrepreneurship and their influence in the process of innovation.

Keywords: Innovation; company; economy; support; competitiveness.

\footnotetext{
${ }^{1}$ Administrador de Empresas, Especialista en Gerencia Financier y Magister en Mercadeo. Docente Investigador de la Escuela Nacional del Deporte. E-mail: luis.david@endeporte.edu.co. ORCID: 0000-0002-5326-7353

${ }^{2}$ Magister en educación. Docente Investigador de la Escuela Nacional del Deporte. E-mail: natali.cruz@endeporte.edu.co.
} 


\section{Introducción}

En la actualidad, el motor de impulso de la economía es el emprendimiento, situación que se evidencia en varios países, y Colombia no es la excepción. En nuestro país, varias entidades se encargan de capacitar al emprendedor en el proceso de consolidación de su idea de negocio y puesta en marcha de una empresa; la capacitación se enfoca en temas empresariales y el acompañamiento para hacer realidad sus proyectos. Por ello, es importante resaltar en el emprendedor la necesidad de crear e innovar al momento de desarrollar su iniciativa, elementos motores del desarrollo empresarial (Cordoba, 2018). Así mismo, Villegas (2001) reconocen que la creatividad e innovación empresarial son un aporte a la generación de prácticas novedosas y diversas de proyectos empresariales.

La labor de los investigadores -entre 2015 y 2017- consistió en brindar asesoría a emprendedores, por medio las cuales se buscó contribuir a la consolidación de la unidad productiva y, junto con el emprendedor, diligenciar el plan de negocio de la idea que posteriormente se llevaba al mercado. En pro de generar mejoras en temas de competitividad, cada emprendimiento recibía capacitaciones en temas de creatividad e innovación, por medio de talleres enfocados metodológicamente a las necesidades y tamaño de las empresas asesoradas. Sin embargo, en la mayoría de los casos el emprendedor no realizaba la incorporación de lo aprendido a su emprendimiento, generando la necesidad de identificar las posibles causas de este fenómeno.

\section{Metodología}

Inicialmente, se verificó el tamaño de las empresas bajo la referencia de la Ley 590 de 2000, conocida como Ley MiPymes, y sus modificaciones en la Ley 905 de 2004. Estas leyes clasifican a las empresas según la cantidad de empleados vinculados y la cantidad de activos, permitiendo identificar el tamaño de los emprendimientos de la muestra, situando como microempresas a las empresas que tienen hasta 10 trabajadores vinculados y activos inferiores a 501 salarios mínimos mensuales vigentes. Como instrumento de recolección de la información se utilizaron los planes de negocio elaborados en conjunto con los emprendedores en los años 2015, 2016 y 2017, teniendo como método el análisis de contenido.

La informalidad de este tipo de emprendimientos mostraba una población infinita, dificultando determinar una base confiable para la presente investigación. Entonces, se eligió una muestra correspondiente a 372 emprendimientos con los siguientes dos criterios de inclusión: microempresas y poco/nulo componente innovador.

Con la información recolectada, se procedió al análisis en el software QDA MINER LITE, facilitando el análisis de textos y palabras que orientaran la búsqueda de información concluyente. Para que la investigación significara un aporte al conocimiento, y que no sólo se basara en una hipótesis, se tomó como referencia el Índice Global de Creatividad (2015), el cual clasificó a Colombia en el puesto 71 de 139 naciones analizadas (Institute, 2016). Para el 2016, el Îndice de Innovación Global situó al país en el número 68, cayendo 8 puestos con respecto al 2014 (Intelectual, 2017). $\mathrm{Al}$ visualizar un panorama desalentador, se reafirmó la necesidad de conocer a fondo qué ocurría en los emprendimientos frente a la innovación.

\section{La innovación en las microempresas}

La innovación no es un tema exclusivo para las grandes empresas o industrias con vocación tecnológica; se trata de una necesidad, sin importar el tamaño o sector económico de cualquier empresa. Según la Ley 1014 de 2006, el emprendedor es alguien capaz de innovar, entendiendo dicho verbo como la capacidad de generar bienes y servicios de una forma creativa, metódica, ética, responsable y efectiva. Esta definición no responde a los componentes innovadores encontrados en los emprendedores de la muestra. 
La información recolectada permitió un análisis más detallado del uso que estas empresas tienen de la innovación, y los factores que inciden en la no incorporación de un modelo de negocio. En cuanto a ello, toda empresa debe tener un proceso de mejora continua que facilite la innovación, a partir de tres grados de desarrollo de la misma: incremental, radical y transformacional. Los anteriores aspectos se definen de la siguiente manera:

- Innovación incremental: se refiere a pequeños cambios que mejoran lo que ya existe. Es lo mismo que mejora continua (Morales, 2013).

- Innovación radical: se refiere a cambios significativos en lo que ya existe (Morales, 2013).

- Innovación transformacional: se refiere a cambios que transforman completamente lo que ya existe (Morales, 2013).

La observación permitió encontrar que algunos emprendimientos se basan en la transformación de productos ya existentes, mediante adaptaciones, mejoras o actualizaciones, representando el $100 \%$ de los que incorporaban la innovación en grado incremental o en una edad temprana. Como afirma Tello (2014) "la invención mediante imitación les permitiría mejorar la posición relativa de la microempresa sin incurrir en excesivos riesgos y costes, siendo ésta la forma más comúnmente seguida por las micro y pequeñas empresas en países desarrollados” (p. 201).

Al consultar a los emprendedores que generaban innovación a partir de la transformación o mejora de productos, ellos argumentaron que el proceso se basaba en generar productos nuevos o diferentes; la mayoría citaba el caso de Apple y Iphone -error común en la capacitación sobre innovación. La dirección hacia la tecnología o creación de productos impulsa en el microempresario la idea del producto final. El uso de metodologías como el design thinking, enfocados a la generación de prototipos, propician lo anterior.
En otros casos, el emprendedor generaba innovación a partir de llevar productos tradicionales a nuevos mercados que pertenecían a su cultura ancestral, o correspondían al aprendizaje realizado durante la estadía en otro país o región que querían adaptar a su nuevo lugar de residencia, representando un $10 \%$ de los emprendimientos. La introducción de dicho producto al nuevo mercado o región, impulsaba al emprendedor a generar una empresa. Lo anterior, se podría acercar a lo afirmado por Ortega:

La innovación no tiene por qué suponer un elevado coste para la empresa, puesto que las innovaciones pueden surgir de una nueva forma de hacer publicidad o una nueva forma de trabajo que ahorre horas de suministros en la empresa. No necesariamente tiene porqué suponer un producto nuevo o mejorado técnicamente o en diseño, con los costes añadidos que esto supone" (Ortega, 2010, p. 104).

En la Figura 1, se puede observar cómo los emprendedores llevan la innovación a su emprendimiento:

Figura 1. Innovación en grado incremental

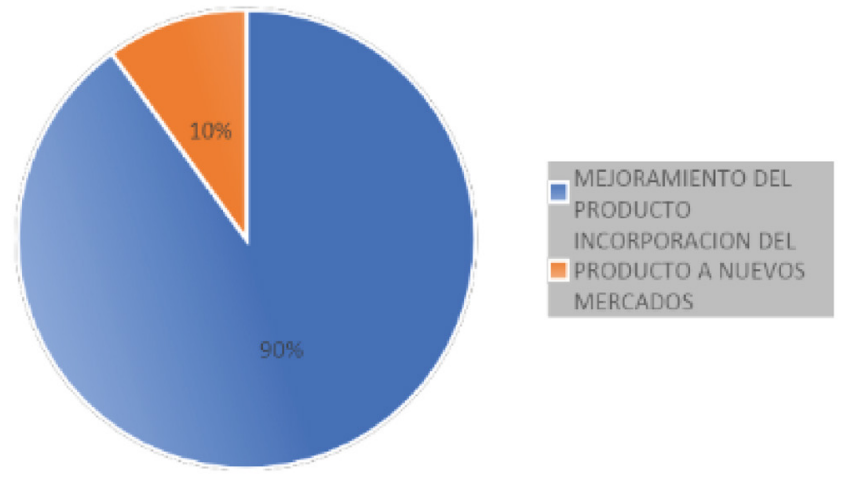

Fuente: Elaboración propiaby 


\section{Motivos para la creación de empresas}

Se observó que el $77,7 \%$ de la muestra tenía como motivo la subsistencia para la creación de su empresa: su emprendimiento es la única fuente de ingresos sin tener claro qué es una empresa o su administración, y limitado solo al concepto de negocio y alejado de temas tan sensibles como la competencia o el mercado. Se debe recalcar que un gran número de empresas creadas no es generalmente proporcional a la competitividad, como lo afirma Callejón y Ortún (2009), los trabajos empíricos muestran que la relación entre la tasa de creación de empresas y la eficiencia económica no es directa.

Según el informe del Global Entrepreneurship Monitor (2015-2016), el 33,3\% de los hombres y mujeres entre 18 y 64 años en Colombia emprendió por necesidad (GEM, 2016), en concordancia con la realidad de la muestra tomada en la presente investigación. La Figura 2 muestra la justificación de los emprendedores para crear empresa, siendo aspectos como la perdida de empleo o la enfermedad, los que conllevan al emprendedor a crear empresa.

\section{Figura 2. Justificación para crear la empresa}

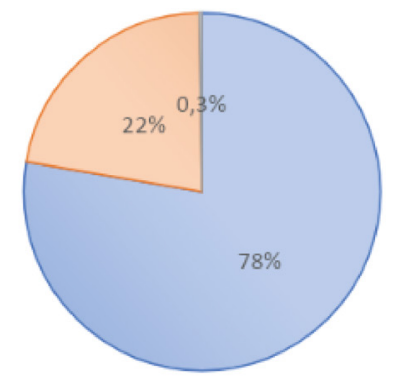

$\square$ NECESIDAD DE INGRESOS $\square$ PERDIDA DEL EMPLEO ENFERMEDAD

Fuente: Elaboración propia

Lo anterior conlleva a la segunda observación: se generan emprendimientos ya validados que facilitan cubrir la necesidad de ingresos económicos. Lo anterior, produce gran concen- tración de emprendimientos en sectores demasiado competitivos. Se evidencian emprendimientos como restaurantes, comidas rápidas o la distribución de alimentos, que alcanzan el 19,4\%; y las cacharrerías, tiendas de barrio o misceláneas con un $26,1 \%$ de la muestra. La facilidad en la creación de estas últimas, por ser ideas previamente existentes en el mercado, va en contravía de la aplicación de la innovación, teniendo en cuenta que toda idea nueva debe tener un proceso de validación en el mercado. Para este caso, se decide arriesgar poco frente al tema, buscando la manera más fácil o rápida de generar ingresos. Lo anterior se puede observar en la Figura 3.

\section{Figura 3. Tipos de emprendimiento}

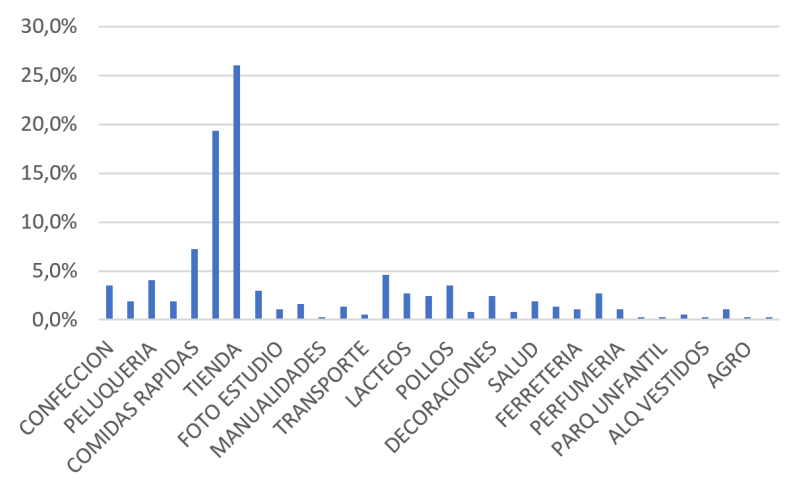

Fuente: Elaboración propia

\section{Propuesta de valor}

En el momento de trabajar con el emprendedor frente a su modelo de negocio, bajo la metodología Canvas, la mayoría presentó la propuesta de valor del producto como innovación; para lo anterior se hace necesario su aclaración: "la propuesta de valor es el factor que hace que un cliente se decante por una u otra empresa; su finalidad es solucionar un problema o satisfacer una necesidad del cliente" (Osterwalder, 2011, p. 20).

El 42,7\% de los emprendedores presentó la calidad del servicio como el valor agregado de su producto, siendo el único factor de innovación. En el mismo sentido, se consideró la entrega a domi- 
cilio, con un $70 \%$ de participación, los pedidos por redes sociales con un $23 \%$, y la atención al cliente con el porcentaje restante.

\section{Figura 4. Propuesta de Valor}

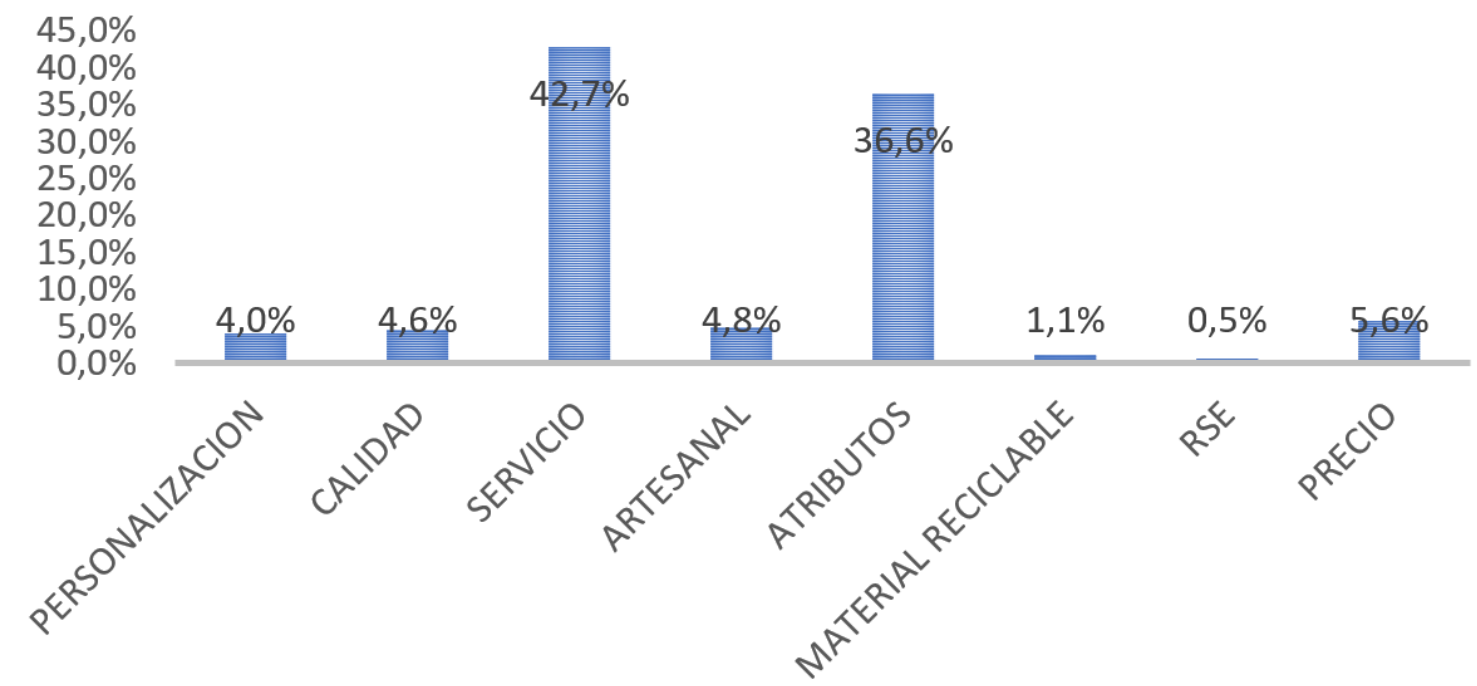

Fuente: Elaboración propia

Para toda empresa, la introducción de un nuevo producto o servicio genera la necesidad de identificar una propuesta de valor coherente a las necesidades del mercado, que sea de valor real y de fácil identificación. En este sentido, la propuesta de valor constituye una serie de ventajas que se debe ofrecer a los clientes. Algunas propuestas de valor pueden ser innovadoras y presentar una oferta nueva, mientras que otras pueden ser parecidas a ofertas ya existentes e incluir alguna característica o atributo adicional (Osterwalder, 2011).

La propuesta de valor debe partir del conocimiento del producto o servicio para identificar qué ofrecer en el mercado, y por consiguiente determinar qué diferenciará el bien o servicio del resto de la competencia. Con lo anterior, se buscaba que el emprendedor identificara la propuesta de valor: el $47 \%$ identificó los atributos del producto, asociando la calidad del mismo o el uso de materiales diferentes frente a su competencia. También se observó que un alto porcentaje de em- prendedores desconocían lo que ofrecían y en qué era posible diferenciarlo, lo cual llevó a que no se pudiera realizar una profundización que permitiera un acompañamiento con el fin de generar innovación en el mismo. Es importante tener en cuenta que un producto se entiende como un artículo, servicio o idea con un conjunto de atributos tangibles o intangibles que satisface a los consumidores y es recibido a cambio de dinero u otra unidad de valor (Kerin, 2009). Así pues, el emprendedor solo percibía su oferta desde los atributos físicos, sin visualizar más allá.

El uso de la metodología Canvas nace de la necesidad de introducir la innovación dentro del modelo de negocio de cada empresa, tratando de ir más allá del producto físico, de la dinámica de los componentes frente a nuevos retos del mercado, y facilitar la innovación empresarial. El empresario debe plasmar lo que es su empresa a partir de nueve componentes que conforman su modelo de negocio. Durante la capacitación, el empresa- 
rio recibió asesoría sobre qué es y qué se pide en cada componente. Para este caso, el emprendedor lo desconoce totalmente y se basa únicamente en el conocimiento empírico obtenido en su trasegar por el mundo empresarial.

Una de las características de estos emprendimientos, es la necesidad que tiene su creador de generar ingresos para su subsistencia. Por ello, en pocos casos correspondían a su desarrollo profesional y la escolaridad de los emprendedores mostraba que el 25,3\% de los empresarios sujetos de la muestra tenía un estudio superior a la primaria o el bachillerato. Dentro de este grupo, encontramos el pequeño porcentaje que incorpora elementos reales de innovación en sus empresas, que no supera el $12 \%$ del total de emprendedores que hacen parte del análisis. Los anteriores resultados son representados en la figura 5.

\section{Figura 5. Nivel de escolaridad}

\section{NIVEL DE ESCOLARIDAD}

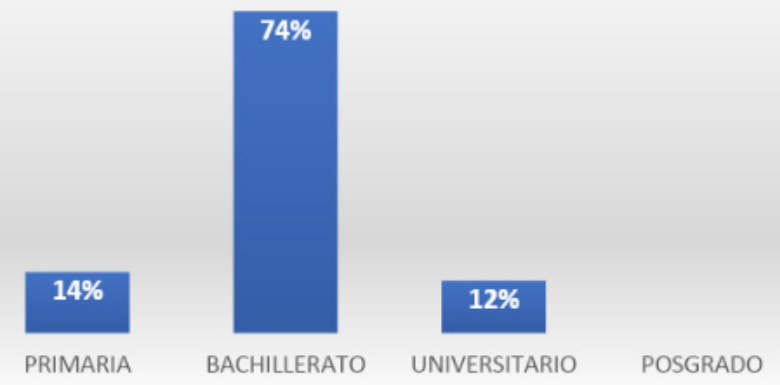

Fuente: Elaboración propia

Desconocer el modelo de negocio dificulta la adaptación a los cambios que exige el mercado. Situaciones como no evaluar las necesidades que moldean el mercado, lleva a que la innovación no tenga un proceso definido.

\section{Ambiente innovador}

La innovación es un proceso constante que se debe realizar en ambientes que propicien el desarrollo de la misma. El emprendedor tiene varios inconvenientes que imposibilitan su participación en dichos ambientes: por un lado, la subsistencia de sus negocios genera la necesidad de pensar en la utilidad o ganancia, garantizando emprendimientos hechos a partir de ideas ya validadas en el mercado, o que se identifican como "seguras" en términos de la inmediatez frente a la consecución de los ingresos, como es el caso del 95\% de la muestra; de esta manera, el desconocimiento de temas empresariales y la inmediatez en las decisiones para emprender les aleja de la innovación.

El acompañamiento al empresario debe realizarse a edades tempranas de la creación de la empresa. El 70\% de los emprendedores nunca habían asistido a una capacitación de emprendimiento, menos para conocer qué es innovación o cuál es su uso. La mayoría de los referidos la identificó como diferenciador o valor agregado del producto o servicio, asociándola a la promoción y mercadeo de los mismos. Por esta razón, el acompañamiento al emprendedor debe influir en la creación de empresas apoyadas en procesos innovadores, que puedan generar impactos en el mercado y no solo emprendimientos de subsistencia. Así pues,

Para que esta situación se pueda producir resulta fundamental que exista una estrecha cooperación entre, por una parte, las universidades y centros de investigación responsables de la generación de nuevo conocimiento científico y tecnológico; y por otra parte, el propio tejido empresarial capaz de explotar los resultados obtenidos en los mercados. Solo cuando se tiene lugar esta cooperación es posible que el conocimiento científico se transforme rápidamente en nuevos productos y servicios o en la mejora de los procesos internos de las empresas" (Calvo, 2011, p. 35).

\section{Discusión de los resultados}

La investigación planteó la premisa que la creatividad y la innovación son los motores del desarrollo empresarial. Lo anterior, se pudo validar en la presente investigación, aunque encontramos que la mayoría de los emprendimientos se 
realizaban por la necesidad de generar ingresos y subsistencia, sin generar desarrollo empresarial, teniendo en cuenta que las empresas entran a participar de mercados ya consolidados o minados de mucha competencia.

Para Villegas (2001), la creatividad e innovación empresarial constituyen un gran aporte a la inventiva y al desarrollo de proyectos. En este caso, se observó que los proyectos de empresa se generaban más por necesidad o subsistencia que, por innovación o creatividad; también se observó que el desarrollo de iniciativas en el sector de las microempresas parte, en muchas ocasiones, de aspectos personales de quienes deciden emprender dichas ideas de negocio. Morales (2013) define la innovación incremental como pequeños cambios al producto, característica de la fase en la que se pudo definir la innovación de los emprendimientos objetos de la muestra. A partir de una visión general de la muestra, se pudo corroborar la anterior hipótesis, identificando cada emprendimiento y cómo incorporaba la innovación.

\section{Conclusiones}

Al culminar el análisis de los testimonios, y el trabajo realizado con cada emprendedor, se puede concluir lo siguiente:

Uno de los mayores motivos de un emprendedor para crear empresa es la subsistencia. Su ingreso al emprendimiento parte de su necesidad de generar un ingreso mensual que responda a sus compromisos; entonces, da inicio a realizar procesos empresariales de una manera empírica, alejado de la academia, donde el crecimiento observado en la apertura de empresas se explica no por oportunidades de mercado o innovaciones, sino por el factor recesión push (Gonzalez, 2018).

La creación de empresas por subsistencia se basa en ideas existentes en el mercado, y cuando el emprendedor incursiona en mercados muy competitivos, la poca incorporación de innovación en el mismo genera herramientas para su sobre- vivencia, lo que ocasiona el cierre de iniciativas en edades tempranas.

Uno de los mayores obstáculos que tiene la innovación en el sector de las microempresas es la poca formación académica del microempresario, que le facilite visualizar su empresa más allá de un ente generador de ingresos. Según Drucker (1986 citado por Villegas, 2001). Si bien en el proceso de creación de empresas se necesitan conocimientos, habilidades, actitudes, aptitudes y atributos, sobre todo se requiere trabajo para lograr la formación y el desarrollo que el proceso exige. Para Drucker, la acción empresarial es una conducta más que un rasgo del carácter, y su base es conceptual y teórica, no meramente intuitiva; por tanto, dicha acción empresarial puede estudiarse y aprenderse.

Otro obstáculo que tiene el microempresario, es la creación de empresas a partir de ideas ya validadas, lo que lleva a que sus competencias y actitudes emprendedoras solo estén enfocadas a este tipo de emprendimientos que le generan ingresos de manera inmediata, evitando la incertidumbre que, en algún momento le puede ocasionar la validación en el mercado de apuestas diferentes.

El emprendedor debe tomar conciencia de la necesidad de incorporar procesos de innovación en su emprendimiento, entendiendo que la misma es una mejora en su competitividad. En este punto es donde es muy necesario un acompañamiento al empresario en la edad temprana de creación de su iniciativa. Aquí un rol importante que pueden hacer las Universidades como parte de organismos aseores a microempresarios.

Las microempresas tienen una gran participación en el total de empresas en el país y son una necesidad para muchas comunidades, generando importante aportes al desarrollo económico; sin embargo, su impacto en términos de innovación es muy bajo y su aporte sigue siendo inferior comparado con las de mayor tamaño. Por tal motivo, se hace necesario impulsar en las mismas niveles 
mayores al de innovación incremental, así como generar más empresarios con pensamientos disruptivos, acercándoles cada vez más a la academia.

El emprendimiento en empresas de tamaño micro, en muchos casos, no tiene una vida más allá de los cinco años -en el mejor de los escenarios. Es posible intuir que este fenómeno se debe a la creación a partir de ideas demasiado validadas en el mercado y que se encuentra una fuerte competencia. Sumado a lo anterior, la poca innovación genera que sea un competidor sin un diferenciador claro para el mercado.

\section{Referencias bibliográficas}

Calvo, J. L. (2011). Innovación: factor clave del éxito empresarial. Bogotá: Ecoe Ediciones.

Cordoba, E. C. (2018). Creatividad e innovacion: Motores de desarrollo empresarial. Lampsakos, 55 - 65.

DNP. (01 de enero de 2006). Empresas en el país. Recuperado de https://www.dnp.gov.co/ programas/desarrollo-empresarial/micropequena-y-mediana-empresa/Paginas/estadisticas-y-estudios.aspx

GEM. (2016). Informe Global Entrepreneurship Monitor.
Gonzalez, G. O. (2018). La microempresa mexicana un asunto de necesidad y no de oportunidad: el caso de colima. Analisis economico , 123 - 142 .

Institute. (2016). Indice global de Creatividad 2015. Suiza: OMPI.

Intelectual. (2017). Índice Mundial de Innovación 2016. Suiza: OMPI.

Kerin, R. H. (2009). Marketing Novena edicion. Mc Graw-Hill Interamericana.

Morales, M. (2013). Adiós a los mitos de la Innovación. Una guía práctica para innovar en América Latina. Innovare.

Ortega, A. (2010). La internacionalizacion de la empresa y la decision de exportar como solucion a la crisis. Revista sociales y juridicas, $88-111$.

Osterwalder, A. \&. (2011). Generación de modelos de negocio. Bogotá: Grupo Planeta.

Tello, S. (2014). Importancia de las micro, peuqeñas y medianas empresas en el desarrollo del pais. LEX, 199 - 218.

Villegas, R. (2001). Innovación empresarial: arte y ciencia en la creación de empresas. Pearson educacion. 